\title{
Function of AURKA protein kinase in the formation of vasculogenic mimicry in triple-negative breast cancer stem cells
}

This article was published in the following Dove Press journal:

OncoTargets and Therapy

13 June 2016

Number of times this article has been viewed

\begin{tabular}{|c|}
\hline Ying Liu ${ }^{1,2, *}$ \\
\hline Baocun Sun ${ }^{1-3, *}$ \\
\hline Tieju Liu ${ }^{1,2, *}$ \\
\hline Xiulan Zhao ${ }^{1,2}$ \\
\hline Xudong Wang ${ }^{3}$ \\
\hline Yanlei Li,2 \\
\hline Jie Meng ${ }^{2}$ \\
\hline Qiang $\mathrm{Gu}^{1,2}$ \\
\hline Fang Liu ${ }^{1,2}$ \\
\hline Xueyi Dong ${ }^{1,2}$ \\
\hline Peimei Liu ${ }^{2}$ \\
\hline Ran Sun ${ }^{2}$ \\
\hline Nan Zhao' \\
\hline $\begin{array}{l}\text { 'Department of Pathology, General } \\
\text { Hospital of Tianjin Medical University, } \\
{ }^{2} \text { Department of Pathology, Tianjin } \\
\text { Medical University, }{ }^{3} \text { Department of } \\
\text { Pathology, Cancer Hospital of Tianjin } \\
\text { Medical University, Tianjin, People's } \\
\text { Republic of China }\end{array}$ \\
\hline
\end{tabular}

Correspondence: Baocun Sun General Hospital and Department of Pathology and Cancer Hospital of Tianjin Medical University, Anshan Road No I54, Tianjin 300052,

People's Republic of China

Tel +86 I3 602। III 92

Fax +8622833368I3

Email sunbaocun@aliyun.com

\begin{abstract}
Tumor cell vasculogenic mimicry (VM), a newly defined pattern of tumor blood supply, signifies the functional plasticity of aggressive cancer cells forming vascular networks. VM and cancer stem cells (CSCs) have been shown to be associated with tumor growth, local invasion, and distant metastasis. In our previous study, CSCs in triple-negative breast cancer were potential to participate in VM formation. In this study, breast CSCs were isolated from the triple-negative breast cancer cell line MDA-MB-231 by using mammosphere culture. Western blotting and reverse transcription polymerase chain reaction showed that mammosphere cells displayed an increased expression of AURKA protein kinase and stem cell marker c-myc and sox2. The VM formation by mammosphere cells was inhibited by AURKA knockdown or the addition of AURKA inhibitor MLN8237. In the meantime, MLN8237 induced the increased E-cadherin and decreased c-myc, sox2, and $\beta$-catenin expressions. The function of AURKA in VM formation was further confirmed using a xenograftmurine model. The results suggested that AURKA protein kinase is involved in VM formation of CSCs and may become a new treatment target in suppressing VM and metastasis of breast cancer.

Keywords: AURKA, cancer stem cells, vasculogenic mimicry, breast cancer
\end{abstract}

\section{Introduction}

Cancer stem cells (CSCs) are cancer cells (found within tumors or hematological cancers) that possess characteristics associated with normal stem cells, specifically the ability to give rise to all cell types found in a particular cancer sample. ${ }^{1}$ Breast CSCs have been identified and isolated from breast cancer tissues and cell lines., ${ }^{2,3}$ Breast cancer is extremely heterogeneous. Perou et $\mathrm{al}^{4}$ classified breast cancer into five categories based on molecular characteristics: luminal subtype A, luminal subtype B/C, normal breast-like subtype, HER2 overexpression subtype, and basal-like breast cancer. Basal-like breast cancer is mainly considered as triple negative (TN), in which estrogen-receptor, progesterone-receptor, and human epidermal growth factor receptor-2 are negative or absent. Furthermore, TN breast cancer is considered as an independent clinical category with extreme malignancy and invasion; this type of cancer also often relapses after a short amount of time, metastasizes to the viscera and the central nervous system, lacks effective therapies and clinical treatment, and causes high death rate. ${ }^{5}$

Tumor cell vasculogenic mimicry (VM), a newly defined pattern of tumor blood supply, describes the functional plasticity of aggressive cancer cells forming vascular networks. Tumor cells develop morphological changes in a specific microenvironment and then form circulatory channels without vascular endothelial cells. As such, blood flows in specific VM channels. ${ }^{6}$ Tumor cells gain blood supply by VM and promote tumor growth, invasion, and metastasis. Tumors exhibiting VM exhibit aggressive

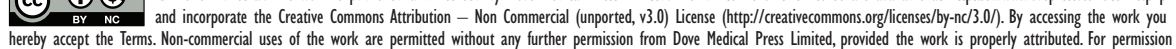
for commercial use of this work, please see paragraphs 4.2 and 5 of our Terms (https://www.dovepress.com/terms.php).
s. 
behavior and increased metastatic ability. ${ }^{7}$ Our previous study $^{8}$ showed that CSCs were able to participate in VM formation and that CSC subpopulation inside TN breast cancer was able to organize VM for which transdifferentiative capacity of CSCs might be needed.

The $A U R K A$ gene is located at chromosome 20q13.2 and encodes serine-threonine kinase, which is composed of 403 amino acids and has vital cellular functions in mitosis. AURKA is considered as an oncogene and plays important roles in the development of breast CSCs by inducing epithelialmesenchymal transition (EMT). ${ }^{9}$ AURKA gene amplification is a common genetic aberration in breast cancer, especially in TN tumors. ${ }^{10}$ Given that both AURKA and VM formation could promote breast cancer invasion and metastasis, the relationship of AURKA and VM in TN breast cancer remains unknown. This study aimed to demonstrate the potential contribution of AURKA to VM in TN breast cancer.

\section{Materials and methods}

\section{Cell culture and isolation of breast CSCs}

The human breast cancer cell lines MDA-MB-231, Hs578T, and MCF-7 were obtained from the American Type Culture Collection (Manassas, VA, USA). This study did not use human tissues or the primary cultured tumor cells, only cell lines were used, thus, such permission was not required, according to General Hospital of Tianjin Medical University review board. These cells were cultured in Dulbecco's Modified Eagle's Medium (Sigma-Aldrich Co., St Louis, MO, USA) supplemented with $10 \%$ fetal bovine serum, 100 units $/ \mathrm{mL}$ penicillin, and $100 \mathrm{mg} / \mathrm{mL}$ streptomycin (Thermo Fisher Scientific, Waltham, MA, USA) in a humidified $5 \% \mathrm{CO}_{2}$ incubator at $37^{\circ} \mathrm{C}$. At the logarithmic growth phase, the MDA-MB-231 or $\mathrm{Hs} 578 \mathrm{~T}$ cells were digested with $0.25 \%$ trypsin and then seeded at $1 \times 10^{5}$ into six-well, ultralow adherent plates covered with poly 2-hydroxyethyl methacrylate (Sigma-Aldrich). Each well also contained $2 \mathrm{~mL}$ of serum-free suspension medium or Dulbecco's Modified Eagle's Medium/F12 (1:1; Thermo Fisher Scientific) supplemented with 2\% B27 (Thermo Fisher Scientific), $0.5 \%$ epidermal growth factor (Pepro Tech; Rocky Hill, NJ, USA), and $0.5 \%$ basic fibroblast growth factor (Pepro Tech). Cell growth was daily observed under an inverted microscope (Nikon USA, Garden City, NY, USA).

\section{Reverse transcription polymerase chain reaction analysis}

To assess the expression levels of c-myc, sox2, E-cadherin, and $\beta$-catenin, we extracted total RNA from the cell lines by using Trizol reagent (Thermo Fisher Scientific) according to the manufacturer's instructions. Polymerase chain reaction was designed to amplify specific mRNAs by using published sequences. The primer sequences were listed as follows: c-myc sense 5'-TACCCTCTCAACGACAGCAG-3', antisense $5^{\prime}$-TCTTGACATTCTCCTCGGTG-3', Sox 2 sense 5'-GGGAAATGGAGGGGTGCAAAAGAGG-3', antisense 5'-TTGCGTGAGTGTGGATGGGATTGGTG- $3^{\prime}, \beta$-catenin sense $5^{\prime}$-AAGGTCTGAGGAGCAGCTTC-3', antisense 5'-TGGACCATAACTGCAGCCTT-3', E-cadherin sense 5'-GTCACTGACACCAACGATAATCCT-3', antisense 5' TTTCAGTGTGGTGATTACGACGTTA- $3^{\prime}, \alpha$-catenin sense 5'-GCTGCTCTCCAACACAGTCA-3', antisense 5'-TGTCATACCAGGAAATGAGCTTG-3', Twist1 sense 5'-GCUGCAGGACUCUAAUCCAdTdT-3', antisense 5'CCGGCTCAgTGGaAtCTtCGAACG- ${ }^{\prime}$, Snail sense 5'-CCTGGCCAAGGTCATCCATGAC-3', antisense 5'-UGGAUUAGAGUCCUGCAGCdTdT-3', Vimentin sense 5'-TCGTTCGAGGTTTCGCGTTAGAGAC-3', antisense 5'-CGACTAAAACTCGACCGACTCGCGA-3', OCT-4 sense 5'-CGACCATCTGCCGCTTTGAG-3', antisense 5'-CCCCCTGTCCCCCATTCCTA-3', NANOG sense, 5'-ACGGTACCTAACATGAGTGTGGATCCAG-3', antisense 5'-TAACTCGAGATCTTCACACGTCTTCAGG-3', $\gamma$-catenin sense $5^{\prime}$-TGTATCTTATGGTACTGTAACTG- $3^{\prime}$, antisense $5^{\prime}$-CTTTATGTTTTTGGCGTCTTCCA-3', glyceraldehyde-3-phosphate dehydrogenase sense 5'-CCTGGCCAAGGTCATCCATGAC-3', antisense 5'-TGTCATACCAGGAAATGAGCTTG-3'.

\section{Western blot analysis}

Cells were collected and lysed. The protein concentration was then determined, and lysates were resolved by sodium dodecyl sulfate-polyacrylamide gel electrophoresis and transferred onto polyvinylidene difluoride membranes (EMD Millipore, Billerica, MA, USA). Blots were blocked with 5\% milk/Tris-Buffered Saline and Tween 20 and then incubated with primary monoclonal antibodies (AURKA, 1:500; c-myc, 1:1,000; sox2, 1:200; VE-cadherin, 1:400; $\beta$-catenin, 1:500; and E-cadherin, 1:500 [Santa Cruz Biotechnology Inc., Dallas, TX, USA]) at $4^{\circ} \mathrm{C}$ overnight and then with secondary antibodies (1:2,000; Santa Cruz Biotechnology Inc., Dallas, TX, USA). Blots were developed using a chemiluminescence detection kit (Amersham Pharmacia Biotech, Piscataway, NJ, USA). Monoclonal $\beta$-actin antibody (1:2,000; Santa Cruz Biotechnology Inc.) was used as a control treatment. All the experiments were repeated thrice and provided reproducible results.

\section{Three dimensional cultures}

Cancer cells were seeded into six-well plates mixed with Matrigel (Collaborative Biomedical, Bedford, MA, USA). A regular 
medium was added to the wells during incubation, and threedimensional (3D) cultures were photographed using a phase contrast microscope (Nikon USA, Garden City, NY, USA).

\section{Cell viability assay with MLN8237}

MDA-MB-231 cells in the logarithmic growth phase were treated with MLN8237 $(10,20$, and $40 \mu \mathrm{M}$ in a final formulation of 10\% 2-hydroxypropyl-cyclodextrin). Cell viability was observed at 24, 48, and 72 hours under an inverted microscope.

\section{Xenograft murine model}

Nonobese diabetic/severe combined immunodeficient female mice (aged 4-5 weeks) were purchased from the Animal Institute of China Academy of Medical Science, Beijing, People's Republic of China. All the animal studies were conducted according to protocols approved by the Ethical Committee of Tianjin Medical University, People's Republic of China, and all the animals were kept according to the Animal Care Guidelines and housed in 12 hours light/dark conditions with free access to food and water. Breast CSCs were collected and resuspended in Dulbecco's Modified Eagle's Medium/ F12 (1:1; Thermo Fisher Scientific). Cells $\left(1 \times 10^{5}\right)$ were inoculated subcutaneously into the right flank of each mouse. The mice were visually examined daily. The mice were then divided into two groups (ten mice in each group) when the tumor volumes could be measured (or 2 weeks after the injection). These mice were subcutaneously injected with $100 \mu \mathrm{L}$ of 10\% 2-hydroxypropyl-cyclodextrin (Sigma-Aldrich)/1\% sodium bicarbonate or MLN8237 (Selleck Chemicals, Houston, TX, USA) (10 mg/kg in a final formulation of $10 \%$ 2-hydroxypropyl-cyclodextrin/1\% sodium bicarbonate) for 10 consecutive days. The maximum tolerated dose of MLN8237 in the majority of mouse strains was $\sim 20 \mathrm{mg} / \mathrm{kg}$ twice a day ( $40 \mathrm{mg} / \mathrm{kg}$ per day). The size of xenograft tumors was measured daily with a caliper throughout the study. Tumor volumes were calculated using the following formula: length $\times$ width $^{2} \times 0.5$. The mice were sacrificed after the treatment was completed. The xenograft tumors were collected from mice, fixed in 4\% formalin, and embedded in paraffin for immunohistochemical and histochemical double-staining.

\section{Immunohistochemical and histochemical double-staining methods}

Tissue sections $(4 \mu \mathrm{m})$ were deparaffinized and hydrated using standard procedures. Immunostaining was performed using a Super Sensitive S-P IHC kit (Thermo Fisher Scientific). Anti- $\beta$-catenin, anti-c-myc, anti-sox2, anti-E-cadherin, and anti-CD31 (Beijing Zhongshan Biotechnology Limited
Company, Peking, People's Republic of China) antibodies were applied to the sections at dilution ratios of 1:50, 1:20, $1: 100,1: 200$, and 1:40, respectively. Later, antigens were retrieved. The sections were initially stained using PicTure PV6000 (Zhongshan Chemical Co., Beijing, People's Republic of China) and Elivision Plus (Zhongshan Chemical Co.) and then counterstained with hematoxylin or periodic acid-Schiff. For the negative control treatment, phosphatebuffered saline was used in place of primary antibodies.

\section{Evaluation of c-myc and sox2 staining}

Positive signals of c-myc and sox 2 proteins were located in the nuclei and cytoplasm. At least ten fields in each specimen were randomly selected and examined under high-power magnification, and $>500$ cells were counted to determine the percentage of positive cells. The case wherein the percentage of positive cells was $\geq 20 \%$ was considered positive.

\section{Evaluation of E-cadherin and $\beta$-catenin staining}

Positive signals of E-cadherin and $\beta$-catenin proteins were located in the cytoplasm and cytomembrane. At least ten power fields were chosen per case and $>500$ cells were counted for each power field. The percentage of the staining cells (P) was scored as follows: 0 (staining of $<5 \%$ of cells), 1 (5\%-25\% of cells), 2 (25\%-50\%), 3 (50\%-75\%), and $4(75 \%-100 \%)$. Staining intensity (I) was graded as follows: 0 (no staining), 1 (weak staining), 2 (moderate staining), and 3 (intense staining). Samples in each power field were evaluated for both factors, ie, $\mathrm{P} \times \mathrm{I}$. The scoring of each case was a mean value of chosen power fields. Eventually the sections were graded as follows: scoring $0-2,1+$; scoring $2-5,2+$; scoring 5-8, 3+; scoring $>8,4+$.

\section{Lentiviral transduction of shRNA AURKA plasmid}

Vector psi-LVRU6GP (GeneCopoeia; Rockville, MD, USA) was used for AURKA silencing in mammosphere cells (MCs). The shRNA target sequences for AURKA were (5'-GGAGTTCTTTGCTATGAATTT-3'). Lentiviruses were produced by transient transfection of $293 \mathrm{~T}$ cells with the plasmids, and lentiviral supernatants were collected 48 hours posttransfection and centrifuged at $500 \times g$ for 10 minutes in order to remove cell debris. Following centrifugation, the supernatant was filtered through $0.45 \mu \mathrm{m}$ polyethersulfone low protein-binding filters. Then the virus suspension was diluted in a complete medium or mammosphere culture medium with polybrene at a final concentration of $8 \mu \mathrm{g} / \mathrm{mL}$ (Sigma-Aldrich) was used to infect the target cells. 


\section{Statistical analysis}

Data were analyzed using SPSS 16.0 (SPSS Inc., Chicago, IL, USA). $P<0.05$ (two-sided) was considered as statistically significant.

\section{Results}

\section{MDA-MB-23 I cells displayed AURKA overexpression}

Western blot was performed to characterize the expression of AURKA protein kinase in MDA-MB-231 cells and MCF-7 cells (Figure 1A). The protein expression levels of AURKA in MDA-MB-231 cells were significantly higher compared with those of the MCF-7 cells.

\section{Breast CSCs are isolated from the TN breast cancer cell line MDA-MB-23I in serum-free suspension culture}

Figure 1B shows MDA-MB-231 cells cultured in Dulbecco's Modified Eagle's Medium supplemented with $10 \%$ fetal bovine serum. Then the serum-free suspension culture was used to isolate breast CSCs subpopulation from MDAMB-231. MDA-MB-231 cells were seeded in six-well, ultralow adherent plates containing a serum-free medium. Bulk cells gradually underwent apoptosis (Figure 1C), and a few cells survived but exhibited a suspended growth pattern. After 72 hours, the cells started to divide into mammospheres, which were compacted with several to dozens of cells.
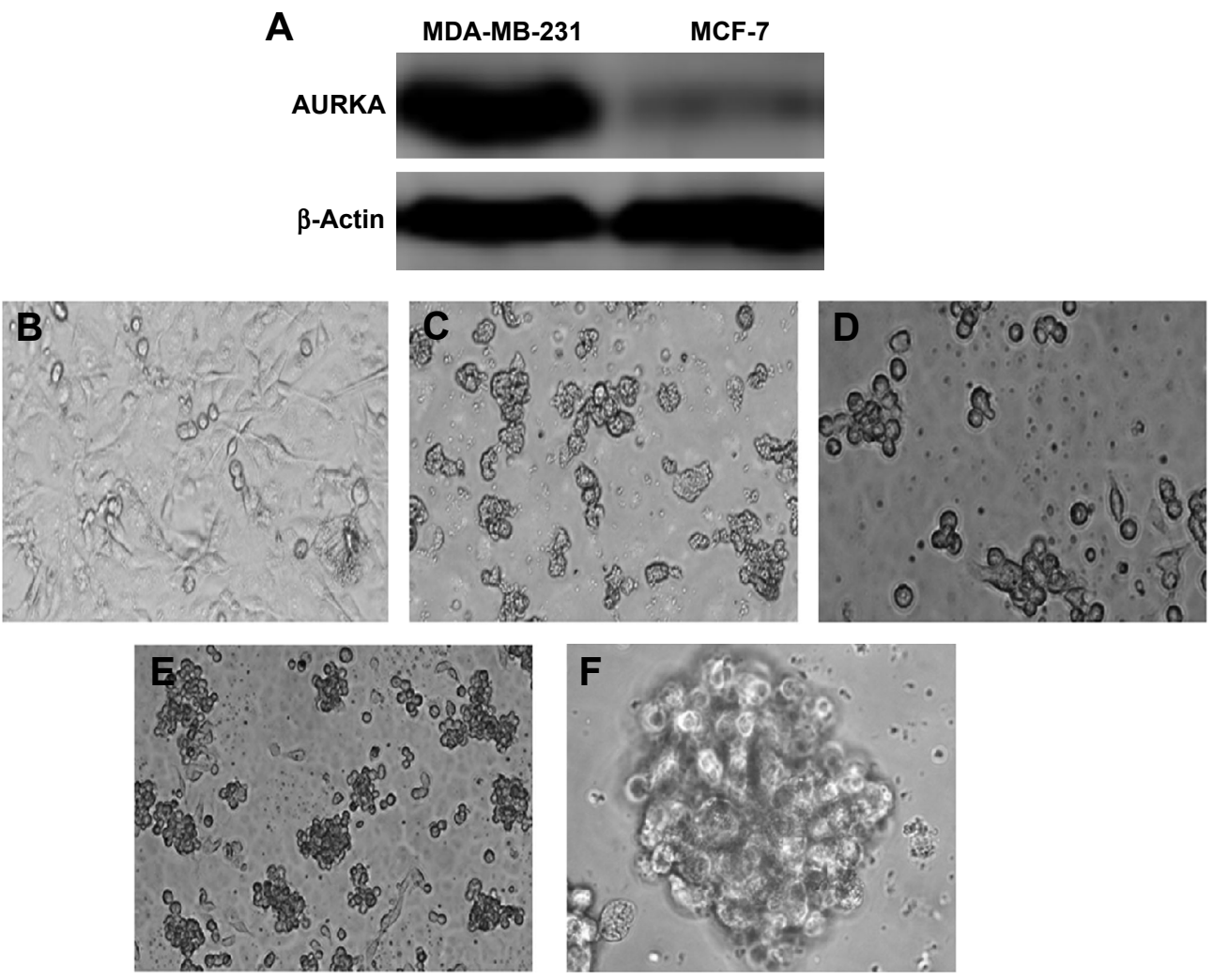

G

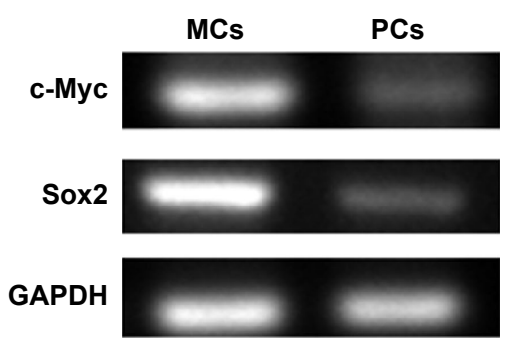

H

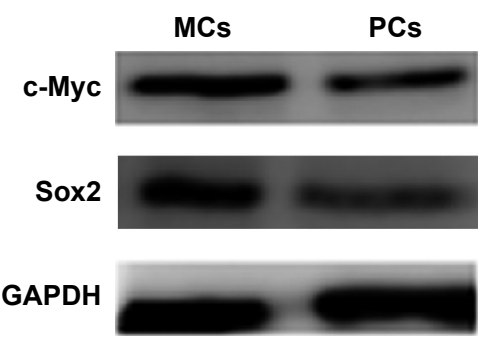

Figure I MDA-MB-23I cells displayed AURKA overexpression and mammospheres culture cells of MDA-MB-23I showed CSCs characteristics.

Notes: (A) MDA-MB-23I cells displayed overexpression of AURKA compared with MCF-7. (B) MDA-MB-23I cells were cultured in regular medium. (C and D) MDAMB-23I cells were seeded into six-well, ultralow adherent plates with serum-free medium; mammospheres were compacted with several to dozens of cells after 72 hours in culture with serum-free medium. (E and F) Mammosphere reproduction was terminated after 10-15 days of proliferation. Mammospheres were large and round or orbicular ovate. Cells were connected tightly, and the margins between these cells were undistinguishable. (G and $\mathbf{H})$ Mammosphere culture cells of MDA-MB-23I showed CSC characteristics. Expression levels of c-myc and sox2 in MCs was higher than those in parental MDA-MB-23I cells (PCs) by RT-PCR (G) and Western blotting (H). Magnification (B and E) $\times 100$; (C and D) $\times 200 ;(\mathbf{F}) \times 400$.

Abbreviations: CSC, cancer stem cell; MCs, mammosphere cells; RT-PCR, reverse transcriptase polymerase chain reaction. 
Incipient mammospheres were small amorphous loose structures. Dividing cells were observed under an inverted phase contrast microscope (Figure 1D). The reproduction of mammospheres was terminated after 10-15 days of cell proliferation. The resulting mammospheres were round or orbicular-ovate in shape. The cells were also tightly connected, and the margins between these cells were undistinguishable (Figure 1E and F).

Reverse transcription polymerase chain reaction and Western blot analyses were performed to examine the expression levels of CSC-related genes c-myc and sox2 in MCs and their parental cells (Figure $1 \mathrm{G}$ and $\mathrm{H}$ ). The relative expression levels of $c-m y c$ and sox 2 in the MCs were significantly elevated than those in parental cell line MDA-MB-231, suggesting that the mammospheres isolated from the $\mathrm{TN}$ breast cancer cell line MDA-MB-231 displayed the characteristics of CSCs.

\section{MCs with CSC characteristics displayed AURKA overexpression}

Western blot was performed to characterize the expression of AURKA protein kinase in MCs and their parental MDAMB-231 cells (Figure 2A). The protein expression levels of

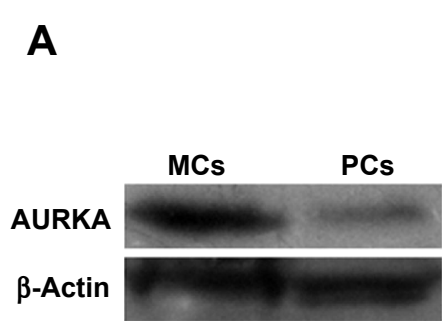

D

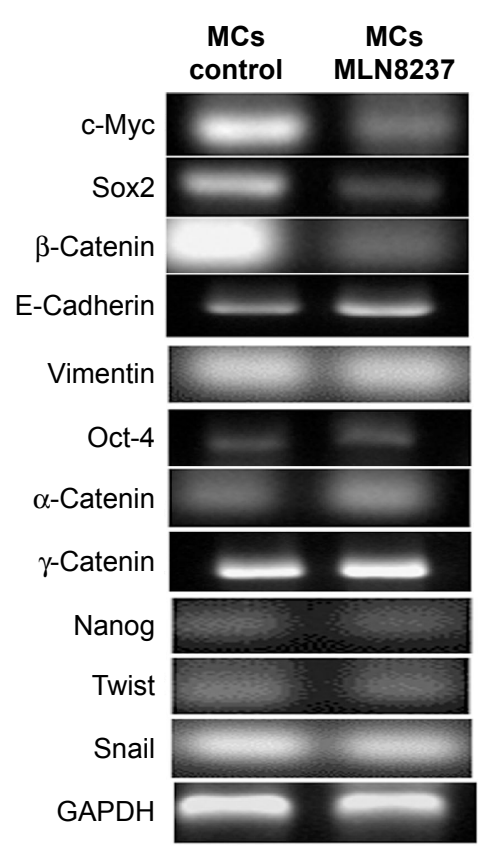

B

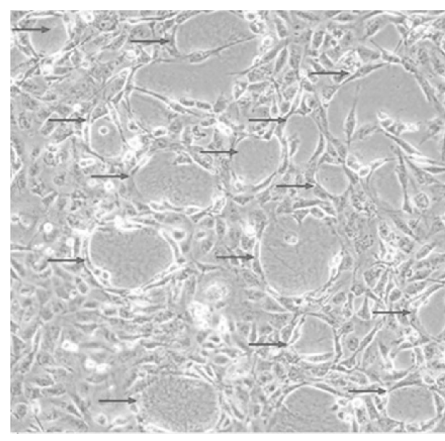

E
C

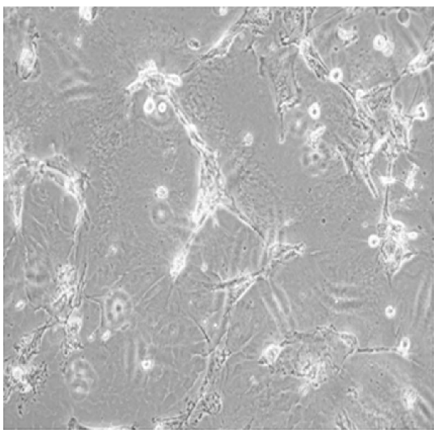

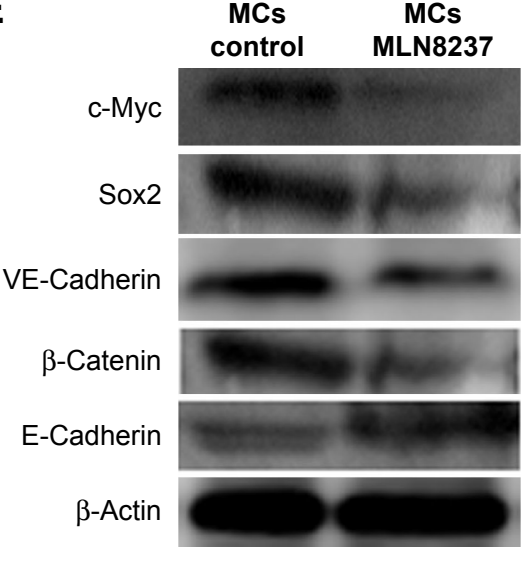

$\mathbf{F}$

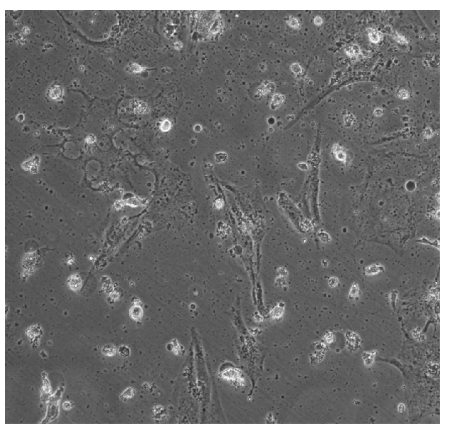

Figure 2 AURKA inhibitor inhibited VM formation of mammosphere cells.

Notes: (A) Expression of AURKA protein kinase in parental MDA-MB-23I cells (PCs) and MCs by Western blot; (B) MCs formed VM channels after 48 hours in 3D Matrigel culture (arrows); (C) VM formation was inhibited by the treatment of AURKA protein kinase inhibitors; (D) RT-PCR showed the decreased mRNA expression levels of c-myc, sox2, and $\beta$-catenin as well as the increased E-cadherin expression by the treatment of AURKA inhibitor MLN8237; there were no differences of the relative expression levels of vimentin, Snail, twist I, Oct4, $\alpha$-catenin, $\gamma$-catenin, and Nanog in MCs after the addition of AURKA protein kinase inhibitor; (E) Western blot showed the decreased protein expression levels of c-myc, sox2, $\beta$-catenin, and VE-cadherin as well as the increased E-cadherin expression by the treatment of AURKA inhibitor MLN8237; and (F) AURKA inhibitor inhibited the growth of MDA-MB-23I cells in vitro culture. Magnification $\times 200$ (B, C, and F).

Abbreviations: 3D, three-dimensional; MCs, mammosphere cells; PC, parental cell; RT-PCR, reverse transcriptase polymerase chain reaction; VM, vasculogenic mimicry. 
AURKA in MCs were significantly increased compared with those of the parental cells.

\section{AURKA inhibitor inhibited VM formation and VE-cadherin expression in MCs}

The vasculogenic capacity of MCs was observed in three dimensional (3D) culture. MCs with CSC characteristics presented spindle or polygonal shape and stretched parapodium after 24 hours when these cells were plated in Matrigel; these cells then formed distinctly characterized microvascular channels after 48 hours (Figure 2B). After AURKA protein kinase inhibitor MLN8237 was added to the 3D culture, MCs aggregated and tubular structure formation was evidently impeded (Figure 2C). In the meantime, VM marker VE-cadherin expression was decreased following AURKA inhibition (Figure 2D). Therefore, our results suggested that AURKA may play a key role in VM formation of MCs.

\section{Effects of AURKA inhibitor on the expression levels of c-myc, sox $2, \alpha, \beta$, $\gamma$-catenin, E-cadherin, vimentin, Snail, twist I, Oct4, and Nanog}

The expression levels of c-myc, sox2, E-cadherin, and $\beta$-catenin are shown in Figure $2 \mathrm{D}$ and E. Following the addition of AURKA protein kinase inhibitor to the MCs, the relative mRNA and protein expression levels of c-myc, sox 2 , and $\beta$-catenin in MCs were significantly decreased and those of E-cadherin were significantly increased by reverse transcription polymerase chain reaction and Western blot assay (Figure 2D and E). However, there were no differences of the relative expression levels of vimentin, Snail, twist1, Oct4, $\alpha, \gamma$-catenin and Nanog in MCs after the addition of AURKA protein kinase inhibitor (Figure 2D).

\section{AURKA inhibitor inhibited growth of MDA-MB-23I cells in vitro culture}

AURKA protein kinase inhibitor MLN8237 with the concentrations of 10,20 , and $40 \mu \mathrm{M}$ was added to the cell culture for 24, 48, and 72 hours, respectively, for the assessment of cell viability. By counting live cells (Figure $2 \mathrm{~F}$ ), cell viability in comparison with the control (untreated cells) was plotted against the doses and the times (Figure 3A). As shown, no significant effects were noticed until 24 hours of incubation. After 48 hours the effects became obvious. Cell viability was also decreased with an increased treatment dose (Figure 3A). This result suggested that MLN8237 inhibited the growth of cancer cells and that there was a dose- and time-dependent effect for MLN8237 treatment.

\section{Influence of the AURKA protein kinase inhibitor MLN8237 on the growth of xenograft murine model}

MLN8237 was subcutaneously injected to nonobese diabetic/ severe combined immunodeficient female mice in order to detect the effect of AURKA protein kinase inhibitor on the growth of xenograft murine model. Tumor growth curve was drawn on the basis of the measured tumor volume (Figure 3B and C). After the cells were inoculated subcutaneously, the mice were treated with MLN8237 for 2 weeks. The tumor growth rate in the treatment group decreased after 4 days and was found to be smaller than that of the control group after 7 days. The tumor growth curve also showed that the tumor growth rate in the treatment group was slower than that of the control group. This result suggested that the MLN8237 inhibited the growth of xenograft murine model.

\section{Effects of AURKA inhibitor on the formation of $\mathrm{VM}$ and the expression levels of c-myc, sox2, $\beta$-catenin, and E-cadherin in vivo}

In our previous study, CD31 and periodic acid-Schiff histochemical and immunohistochemical double-staining were performed to identify VM in breast cancer. ${ }^{8,11,12}$ In this experiment, VM was characterized by CD31-negative and periodic acid-Schiff-positive vascular-like patterns containing red blood cells (Figure 3D), which were formed by breast cancer cells. The result showed that the control group exhibited $\mathrm{VM}$ at a greater extent than the treatment group $\left(\chi^{2}=6.67, P=0.03\right)$ (Table 1). Among the ten mice in the control group, five (50\%) exhibited VM; by comparison, the mice did not have VM formation when MLN8237 treatment was given (Figure 3D). Although the expressions of Twist and vimentin exhibited no significant differences between the control group and the treatment group, the expressions of c-myc, sox $2, \beta$-catenin, and E-cadherin were significantly different (Figure 4A). Among the ten mice, four $(40 \%)$ were c-myc positive in the control group; by contrast, the mice in the treatment group were c-myc negative $\left(\chi^{2}=8.57, P=0.01\right)$ (Table 2$)$. Similar to VM, c-myc expression was dramatically decreased following MLN8237 treatment in vivo. Likewise, four (40\%) of ten mice in the control group and one (10\%) of ten mice in the treatment group were sox 2 positive. This result indicated that sox 2 expression was decreased in the treatment group than in the control group $\left(\chi^{2}=5.50, P=0.02\right)$ (Table 3$)$. For $\beta$-catenin expression, the following median values were 
A

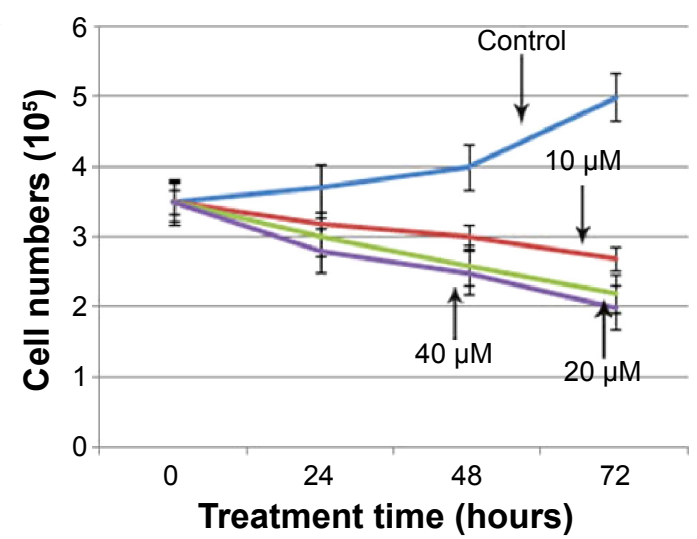

B

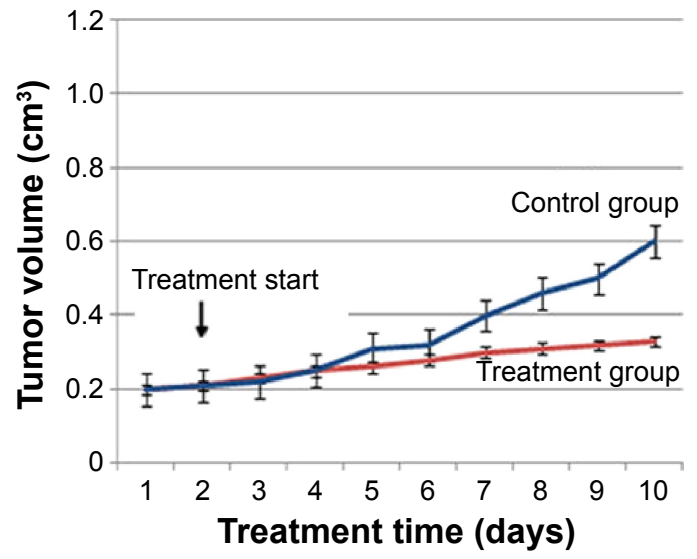

C

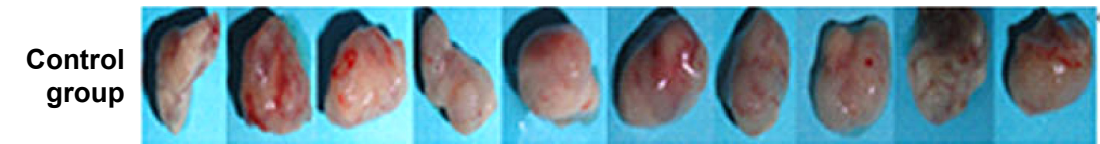

Treatment group

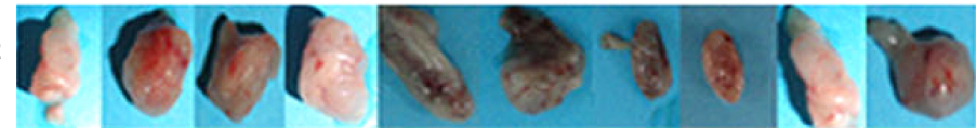

D
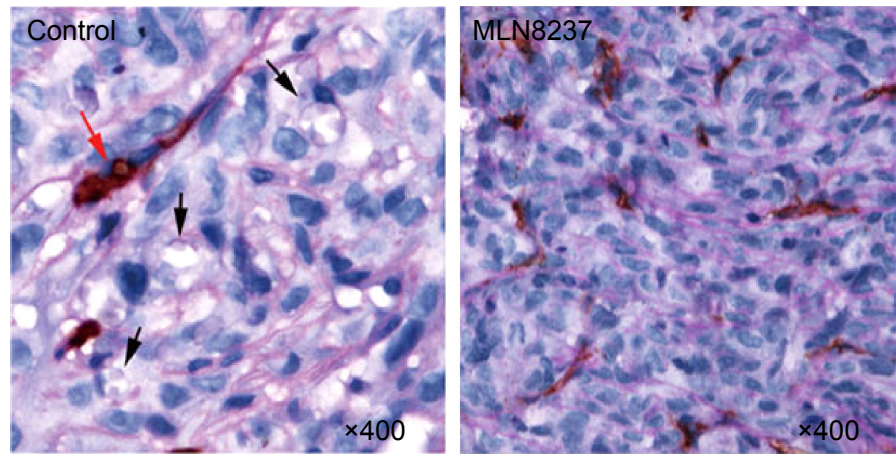

Figure 3 AURKA inhibitor inhibited the growth of xenograft murine model.

Notes: (A) Cell viability curve was plotted on the basis of the measured cell numbers. (B and C) Tumor growth curve in MC control xenografts and AURKA inhibitor treatment xenografts. (D) AURKA inhibitor inhibited VM formation in MCs xenografts. VM (black arrows) in MC control xenografts was identified by CD3I and PAS doublestaining, and red arrow indicates typical blood vessel; and on the contrary, the mice did not show VM formation when the MLN8237 treatment was given.

Abbreviations: MCs, mammosphere cells; PAS, periodic acid-Schiff; VM, vasculogenic mimicry.

obtained: $3.90 \pm 1.60$ in the control group and $2.30 \pm 0.68$ in the treatment group. This result indicated that $\beta$-catenin expression was decreased in the treatment group than in the control group ( $t=2.92, P=0.01$ ) (Table 4). For E-cadherin expression, the following median values were obtained: $2.10 \pm 0.74$ in the control group and $3.80 \pm 1.03$ in the treatment group. Contrary to the expressions of c-myc, sox 2 , and $\beta$-catenin, the expression level of E-cadherin was greater

Table I VM in human breast cancer xenografts in nude mice

\begin{tabular}{llllll}
\hline Group & VM & & \multirow{2}{*}{$\chi^{2}$} & P-value \\
\cline { 2 - 3 } & Positive & Negative & & \\
\hline Control & 5 & 5 & 6.67 & 0.03 \\
Administration & 0 & 10 & & \\
\hline
\end{tabular}

Abbreviation: VM, vasculogenic mimicry. in the treatment group than in the control group $(t=4.24$, $P=0.00$ ) (Table 5). Therefore, the results suggested that AURKA inhibitor could not only inhibit VM formation and c-myc, sox 2 , and $\beta$-catenin expressions but also induce epithelial phenotype of cancer cells in vivo.

\section{KD of AURKA inhibited the expression levels of c-myc, sox2, and the formation of $\mathrm{VM}$ in $3 \mathrm{D}$ culture}

We generated stable AURKA KD in MCs using lentiviral shRNA. Western blot analysis revealed that over 90\% KD efficiency at the protein level was achieved by AURKA shRNA compared with the control harboring nontarget shRNA (Figure 4B). Following AURKA KD, the relative mRNA expression levels of c-myc and sox 2 in MCs were 

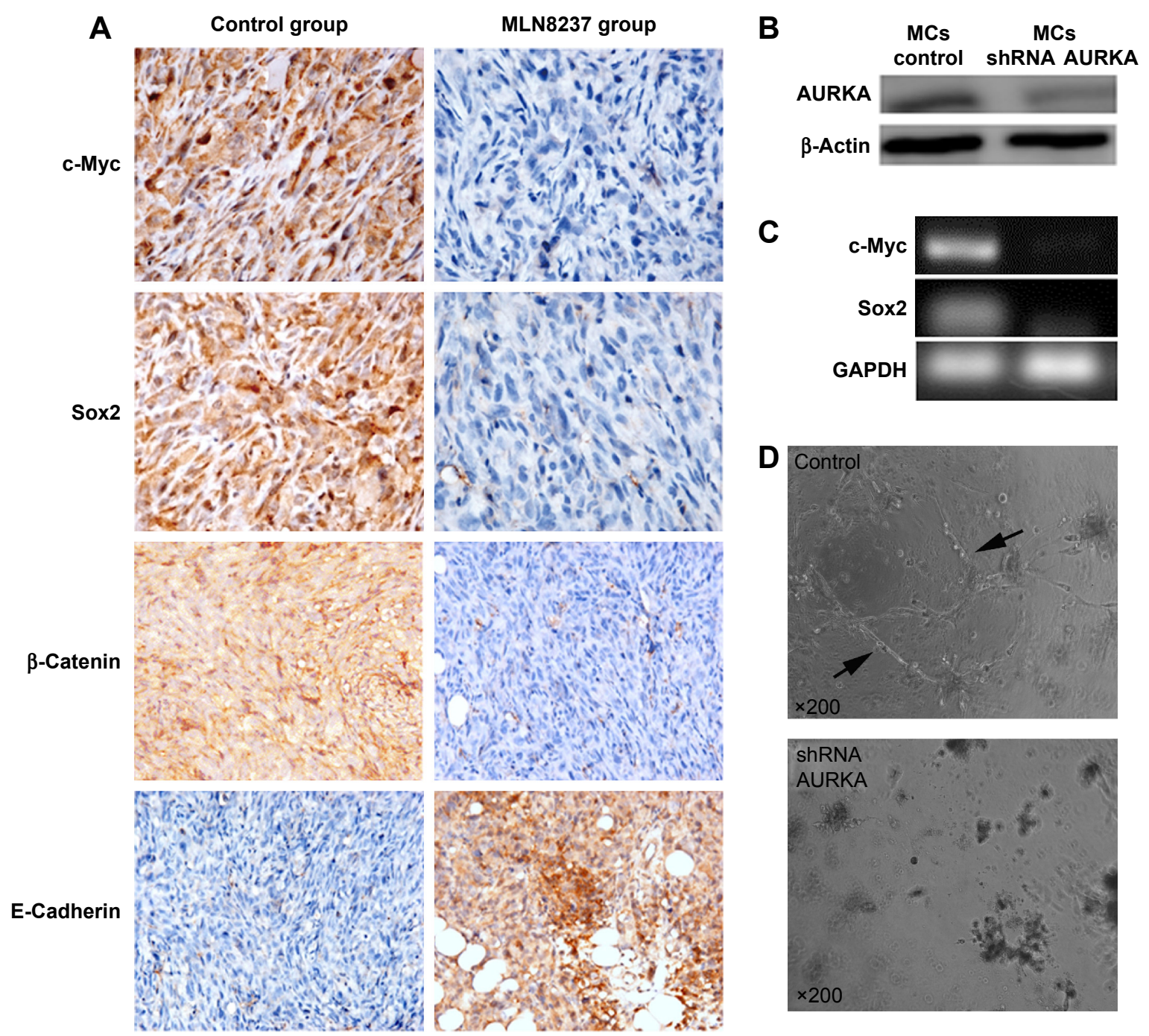

Figure 4 Effects of AURKA inhibitor on the formation of VM and the expression levels of c-myc, sox-2, $\beta$-catenin, and E-cadherin in vivo and plasmid transfection. Notes: (A) IHC showed the decreased expression levels of c-myc, sox2, and $\beta$-catenin as well as the increased E-cadherin expression by the treatment of AURKA inhibitor MLN8237 in vivo (Magnification $\times 400$ ). (B) Western blotting revealed over $90 \%$ knockdown efficiency at the protein level achieved by AURKA shRNA compared with the control harboring nontarget shRNA. (C) The relative mRNA expression levels of c-myc and sox2 in MCs were significantly decreased after the knockdown of AURKA. (D) MC control formed distinctly characterized microvascular channels (arrows) after 72 hours, while AURKA knockdown-induced aggregated cells and tubular structure formation were evidently impeded.

Abbreviations: IHC, immunohistochemistry; MCs, mammosphere cells.

significantly decreased (Figure 4C). MCs showed spindle or polygonal shape and stretched parapodium after 36 hours when these cells were plated in Matrigel; these cells then formed distinctly characterized microvascular channels after 72 hours (Figure 4D). However, after KD of AURKA, MCs aggregated and tubular structure formation was evidently impeded (Figure 4D).

Table 2 c-Myc in human breast cancer xenografts in nude mice

\begin{tabular}{|c|c|c|c|c|}
\hline \multirow[t]{2}{*}{ Group } & \multicolumn{2}{|l|}{ c-Myc } & \multirow[t]{2}{*}{$\chi^{2}$} & \multirow[t]{2}{*}{$P$-value } \\
\hline & Positive & Negative & & \\
\hline Control & 4 & 6 & 8.57 & 0.01 \\
\hline Administration & 0 & 10 & & \\
\hline
\end{tabular}

\section{AURKA KD inhibited VM formation of MCs isolated from other TN breast cancer cells}

In order to demonstrate that AURKA exhibit similar effect on other TN breast cancer cells, the MCs were isolated from Hs578T cells that have been testified as estrogen-receptor-negative, progesterone-receptor-negative,

Table 3 Sox2 in human breast cancer xenografts in nude mice

\begin{tabular}{lllll}
\hline Group & Sox2 & & $\chi^{2}$ & P-value \\
\cline { 2 - 3 } & Positive & Negative & & \\
\hline Control & 4 & 6 & 5.50 & 0.02 \\
Administration & $\mathrm{I}$ & 9 & & \\
\hline
\end{tabular}


Table $4 \beta$-Catenin in human breast cancer xenografts in nude mice

\begin{tabular}{llll}
\hline Group & $\beta$-Catenin & $\boldsymbol{t}$ & $\boldsymbol{P}$-value \\
\hline Control & $3.90 \pm 1.60$ & 2.92 & 0.01 \\
Administration & $2.30 \pm 0.68$ & & \\
\hline
\end{tabular}

Note: Data presented as mean \pm standard deviation.

and HER2 negative. ShRNA AURKA plasmid was used for AURKA protein kinase KD in MCs of Hs578T cells. Western blot was performed to characterize the expression of AURKA in MC control and shRNA AURKA cells (Figure 5A). The protein expression level of AURKA was significantly decreased following AURKA KD in MCs. Meanwhile, the relative mRNA expression levels of c-myc, sox2, and VM marker VE-cadherin in Hs578T MCs were significantly decreased following KD of AURKA (Figure 5B). In Matrigel culture showed that Hs578T MCs formed distinctly characterized microvascular channels after 72 hours (Figure 5C). In contrast, AURKA KD-induced MCs aggregated in Matrigel, and tubular structure formation was evidently inhibited (Figure 5D).

\section{Discussion}

TN breast cancer exhibits distinct molecular phenotypic characteristics and clinically biological behaviors. The lack of treatment targets and drugs is one of the drawbacks that result in poor prognosis of patients with TN breast cancer. As such, specific treatment targets and drugs should be developed to improve prognosis. A considerable amount of studies on medical science transformation have focused on such specific treatment targets and medicines for TN breast cancer.

CSCs are considered as the origin of tumorigenesis and development; these cells can undergo self-renewal and multidirectional differentiation. ${ }^{13,14}$ Al-Hajj et al ${ }^{2}$ initially isolated and identified breast CSCs (accounting for $2 \%$ of the total tumor cell population) and demonstrated the presence of breast CSCs in solid tumors. Ponti et $\mathrm{al}^{3}$ further isolated breast CSCs by using serum-free suspension culture and showed that breast CSCs can survive in serum-free media supplemented with growth factors (eg, epidermal growth factor, basic fibroblast growth factor, and B27). In the present study, serum-free suspension culture was used to isolate

Table 5 E-Cadherin in human breast cancer xenografts in nude mice

\begin{tabular}{llll}
\hline Group & E-Cadherin & $\boldsymbol{t}$ & $\boldsymbol{P}$-value \\
\hline Control & $2.10 \pm 0.74$ & 4.424 & 0.00 \\
Administration & $3.80 \pm 1.03$ & & \\
\hline
\end{tabular}

Note: Data presented as mean \pm standard deviation. breast CSCs as mammospheres, and non-CSCs gradually undergo apoptosis in serum-free cultures. According to our published study, ${ }^{15}$ breast CSC subpopulation enriched by mammosphere culture showed ALDH1 positive by flow cytometry. Therefore, in this study the MCs would represent $\mathrm{ALDH1}^{+}$population. The CSC characteristics of MCs were further testified by detecting the expression levels of stem cell markers, including C-myc and sox2. Our results showed that MCs isolated from MDA-MB-231 cells exhibited characteristics similar to CSCs.

EMT is a multistep morphogenetic process during which epithelial cells downregulate their epithelial properties and upregulate mesenchymal characteristics. ${ }^{16}$ Recent studies report that the induction of EMT in immortalized human mammary epithelial cells results in the acquisition of mesenchymal traits and in the expression of stem-cell markers. The transformed human mammary epithelial cells that have undergone EMT have increased the ability to form mammospheres. ${ }^{17,18}$ The change in E-cadherin expression is a major regulatory mechanism of EMT, and low E-cadherin expression indicates the occurrence of EMT. $\beta$-Catenin is the key component of $\beta$-catenin/Wnt signaling pathway. The accumulation of $\beta$-catenin in cytoplasmic and its subsequent nuclear transfer eventually lead to Wnt target genes such as c-Jun, c-Myc, fibronectin, and cyclinDl activation. Downregulation of E-cadherin expression often results in an increase of $\beta$-catenin, which binds to $\mathrm{T}$ cell factor/lymphoid enhancer factor to participate in transcription regulation. ${ }^{19,20}$ Our previous study suggested that EMT contributed to the formation of tumor VM. ${ }^{21}$ Other studies showed that AURKA could induce EMT and facilitate breast cancer cell metastasis and that AURKA inhibitor could reverse EMT phenotype..$^{22-24}$ The results of this study showed that AURKA inhibitor induced increased E-cadherin and decreased c-myc, sox 2 and $\beta$-catenin expression in MCs in vitro and in vivo, suggesting that AURKA might play an important role in the development of breast CSCs and EMT phenotype.

Maniotis et $\mathrm{al}^{6}$ discovered a new tumor vascular pattern $\mathrm{VM}$, which was independent from tumor vascularization; in this pattern, cancer cells mimic endothelial cells to form extracellular matrix-rich channels that allow blood flow in the circulatory system. VM channels can interconnect with host blood vessels, thereby providing abundant blood supply for tumors. ${ }^{25}$ During the development, multilineage differentiation plasticity is one of the characteristics of embryonic stem cells. Normal tissue-resident stem cells could differentiate into endothelial cells and participate in normal tissue vascularization, which is called transdifferentiation. 
A

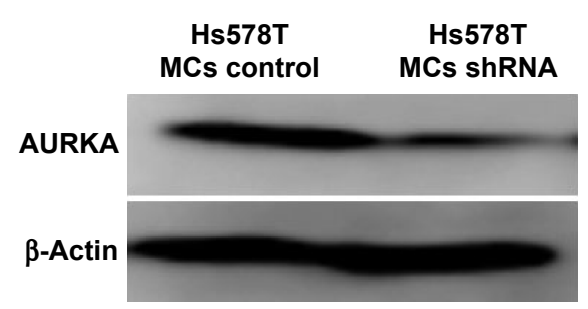

C

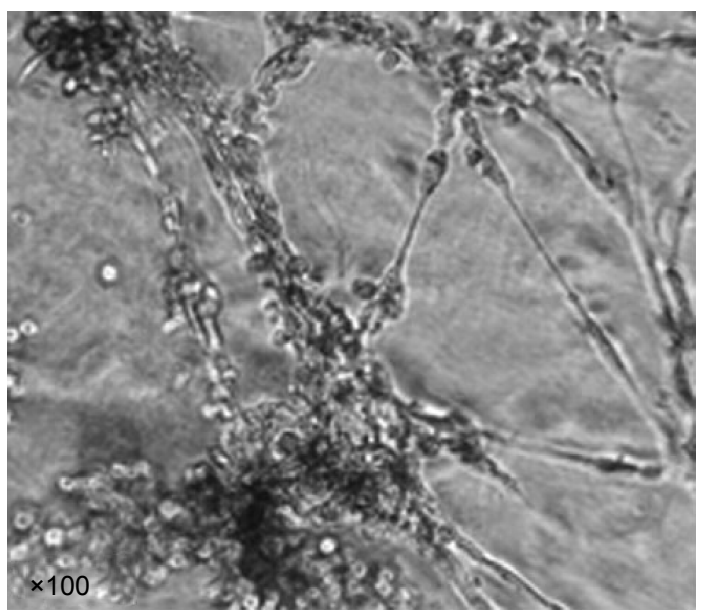

B

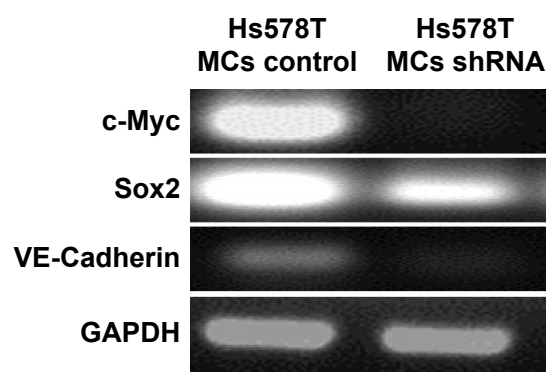

D

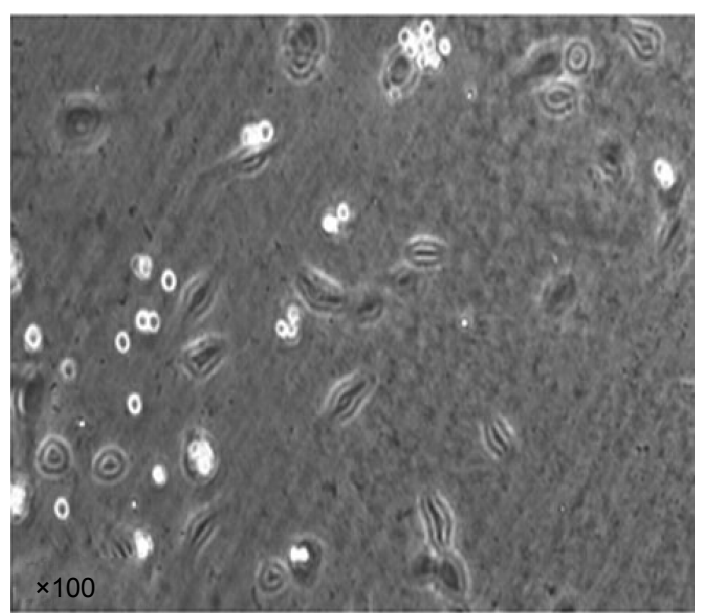

Figure 5 Similar effects of AURKA in Hs578T.

Notes: (A) The protein expression level of AURKA was significantly decreased following AURKA knockdown in MCs of Hs578T. (B) The relative mRNA expression levels of c-myc, sox2, and VM marker VE-cadherin in Hs578T MCs were significantly decreased following AURKA knockdown. (C) Hs578T MCs formed VM channels after 72 hours in 3D Matrigel culture. (D) VM formation in Hs578T MCs was inhibited following AURKA knockdown.

Abbreviations: 3D, three-dimensional; MCs, mammosphere cells; VM, vasculogenic mimicry.

The transdifferentiative capacity and differentiation plasticity of normal stem cells are also common characteristics of CSCs. Yao et $\mathrm{al}^{26}$ proposed a concept of CSC plasticity in which CSCs possess inducible and reversible properties in multipotent differentiation. It refers to the ability of tumor cells to give rise to phenotypically diverse populations including nontumorigenic cancer cells and stromal cells. The research in melanoma, glioma, and renal carcinomas showed that CSCs can transdifferentiate into vascular endothelial phenotype and express angiogenic and vasculogenic markers. In our previous study, ${ }^{8}$ we showed that CSC subpopulation inside TN breast cancer was able to organize VM and therefore played an important role in VM formation. AURKA is an oncogene involved in tumorigenesis and progression. AURKA overexpression is observed in esophageal carcinoma, laryngeal carcinoma, hepatoma, and ovarian cancer. ${ }^{27}$ In the present study, AURKA expression in MCs was significantly higher than that in the parental cells, suggesting that AURKA could be involved in self-renewal and maintenance of breast CSC subpopulation. Therefore, we designed this study to demonstrate the role of AURKA in VM formation of CSCs. In this study, MCs exhibiting characteristics similar to
CSCs could form VM channels in 3D culture. The formation of VM structure was evidently impeded after AURKA KD or MLN8237, a selective AURKA inhibitor, which targets AURKA protein, ${ }^{28,29}$ was added to the $3 \mathrm{D}$ culture. Therefore, this study demonstrates that AURKA protein kinase functions in VM formation of breast CSCs. Although specific regulatory mechanisms remain unclear, AURKA may be considered as a new treatment target to inhibit VM in TN breast cancer.

During the process of VM formation, vessels are directly formed by tumor cells without endothelial cell lining, and tumor cells in VM are prone to defluxion into circulation, thereby inducing distant metastasis. ${ }^{30}$ Our previous study revealed that patients with primary hepatocellular carcinoma exhibiting VM have shorter life span than those without VM. Gastrointestinal tract interstitialoma with positive VM expression is also prone to develop liver metastasis., ${ }^{7,31}$ The traditional treatments of antitumor angiogenesis have focused on vascular endothelial cells. With the discovery of VM in tumors, researchers have been prompted to reconsider the treatment of antitumor VM formation. The treatment that effectively suppresses blood supply provided by VM 
would produce slight toxic effects to normal cells. Thus, this treatment could be safer than chemotherapeutics that elicit adverse cytotoxic effects. ${ }^{32}$ Our results demonstrate that AURKA protein kinase is involved in the development of breast CSCs, EMT, and VM. Hence, AURKA may be considered as a new target treatment to suppress VM and metastasis of breast cancer.

\section{Acknowledgments}

This work was partly supported by a grant from key project of the National Natural Science Foundation of China (No 81230050), the National Natural Science Foundation of China (Nos 81172046, 81173091, and $81201791)$, key project of the Tianjin Natural Science Foundation (No 12JCZDJC23600), the Tianjin Natural Science Foundation (No 12JCYBJC15500), and the Natural Science Foundation of Tianjin Education Commission (No 20120103).

\section{Disclosure}

The authors report no conflicts of interest in this work.

\section{References}

1. Reya T, Morrison SJ, Clarke MF, Weissman IL. Stem cells, cancer, and cancer stem cells. Nature. 2001;414(6859):105-111.

2. Al-Hajj M, Wicha MS, Benito-Hernandez A, Morrison SJ, Clarke MF. Prospective identification of tumorigenic breast cancer cells. Proc Natl Acad Sci U S A. 2003;100(7):3983-3988.

3. Ponti D, Costa A, Zaffaroni N, et al. Isolation and in vitro propagation of tumorigenic breast cancer cells with stem/progenitor cell properties. Cancer Res. 2005;65(13):5506-5511.

4. Perou CM, Sorlie T, Eisen MB, et al. Molecular portraits of human breast tumours. Nature. 2000;406(6797):747-752.

5. O'Shaughnessy J, Osborne C, Pippen JE, et al. Iniparib plus chemotherapy in metastatic triple-negative breast cancer. $N$ Engl J Med. 2011; 364(3):205-214.

6. Maniotis AJ, Folberg R, Hess A, et al. Vascular channel formation by human melanoma cells in vivo and in vitro: vasculogenic mimicry. Am J Pathol. 1999;155(3):739-752.

7. Sun B, Zhang S, Zhang D, et al. Vasculogenic mimicry is associated with high tumor grade, invasion and metastasis, and short survival in patients with hepatocellular carcinoma. Oncol Rep. 2006;16(4):693-698.

8. Liu TJ, Sun BC, Zhao XL, et al. CD133+ cells with cancer stem cell characteristics associates with vasculogenic mimicry in triple-negative breast cancer. Oncogene. 2013;32(5):544-553.

9. Acu ID, Liu T, Suino-Powell K, et al. Coordination of centrosome homeostasis and DNA repair is intact in MCF-7 and disrupted in MDA-MB 231 breast cancer cells. Cancer Res. 2010;70(8):3320-3328.

10. Staff S, Isola J, Jumppanen M, Tanner M. Aurora-A gene is frequently amplified in basal-like breast cancer. Oncol Rep. 2010;23(2): 307-312.

11. Liu T, Sun B, Zhao X, et al. OCT4 expression and vasculogenic mimicry formation positively correlate with poor prognosis in human breast cancer. Int J Mol Sci. 2014;15(11):19634-19649.
12. Liu T, Sun B, Zhao X, et al. HER2/neu expression correlates with vasculogenic mimicry in invasive breast carcinoma. $J$ Cell Mol Med. 2013;17(1):116-122.

13. Dawood S, Shaikh AJ, Buchholz TA, et al. The use of bevacizumab among women with metastatic breast cancer: a survey on clinical practice and the ongoing controversy. Cancer. 2012;118(11):2780-2786.

14. Hwang-Verslues WW, Kuo WH, Chang PH, et al. Multiple lineages of human breast cancer stem/progenitor cells identified by profiling with stem cell markers. PLoS One. 2009;4(12):e8377.

15. Liu T, Sun B, Zhao X, et al. USP44+ cancer stem cell subclones contribute to breast cancer aggressiveness by promoting vasculogenic mimicry. Mol Cancer Ther. 2015;14(9):2121-2131.

16. Gavert N, Ben-Ze'ev A. Epithelial-mesenchymal transition and the invasive potential of tumors. Trends Mol Med. 2008;14(5):199-209.

17. Mani SA, Guo W, Liao MJ, et al. The epithelial-mesenchymal transition generates cells with properties of stem cells. Cell. 2008;133(4): 704-715.

18. Shuang $\mathrm{ZY}, \mathrm{Wu} \mathrm{WC}, \mathrm{Xu}$ J, et al. Transforming growth factorbeta1-induced epithelial-mesenchymal transition generates ALDHpositive cells with stem cell properties in cholangiocarcinoma. Cancer Lett. 2014;354(2):320-328.

19. Tepera SB, McCrea PD, Rosen JM. A beta-catenin survival signal is required for normal lobular development in the mammary gland. $J$ Cell Sci. 2003;116(Pt 6):1137-1149.

20. Pitman M, Emery B, Binder M, Wang S, Butzkueven H, Kilpatrick TJ. LIF receptor signaling modulates neural stem cell renewal. Mol Cell Neurosci. 2004;27(3):255-266.

21. Sun D, Sun B, Liu T, et al. Slug promoted vasculogenic mimicry in hepatocellular carcinoma. J Cell Mol Med. 2013;17(8):1038-1047.

22. Baba Y, Nosho K, Shima K, et al. Aurora-A expression is independently associated with chromosomal instability in colorectal cancer. Neoplasia. 2009;11(5):418-425.

23. Lassmann S, Shen Y, Jutting U, et al. Predictive value of Aurora-A/STK15 expression for late stage epithelial ovarian cancer patients treated by adjuvant chemotherapy. Clin Cancer Res. 2007;13(14):4083-4091.

24. Zhou H, Kuang J, Zhong L, et al. Tumour amplified kinase STK15/ BTAK induces centrosome amplification, aneuploidy and transformation. Nat Gen. 1998;20(2):189-193.

25. Lin $P$, Wang W, Sun BC, et al. Vasculogenic mimicry is a key prognostic factor for laryngeal squamous cell carcinoma: a new pattern of blood supply. Chin Med J. 2012;125(19):3445-3449.

26. Yao XH, Ping YF, Bian XW. Contribution of cancer stem cells to tumor vasculogenic mimicry. Protein Cell. 2011;2(4):266-272.

27. Shi Y, Sun G, Zhao C, Stewart R. Neural stem cell self-renewal. Crit Rev Oncol Hematol. 2008;65(1):43-53.

28. Gorgun G, Calabrese E, Hideshima T, et al. A novel Aurora-A kinase inhibitor MLN8237 induces cytotoxicity and cell-cycle arrest in multiple myeloma. Blood. 2010;115(25):5202-5213.

29. Matulonis UA, Sharma S, Ghamande S, et al. Phase II study of MLN8237 (alisertib), an investigational Aurora A kinase inhibitor, in patients with platinum-resistant or -refractory epithelial ovarian, fallopian tube, or primary peritoneal carcinoma. Gynecol Oncol. 2012;127(1): 63-69.

30. Folberg R, Maniotis AJ. Vasculogenic mimicry. APMIS. 2004;112(7-8): 508-525.

31. Sun B, Qie S, Zhang S, et al. Role and mechanism of vasculogenic mimicry in gastrointestinal stromal tumors. Hum Pathol. 2008; 39(3):444-451.

32. van der Schaft DW, Seftor RE, Seftor EA, et al. Effects of angiogenesis inhibitors on vascular network formation by human endothelial and melanoma cells. J Natl Cancer Inst. 2004;96(19):1473-1477. 


\section{Publish your work in this journal}

OncoTargets and Therapy is an international, peer-reviewed, open access journal focusing on the pathological basis of all cancers, potential targets for therapy and treatment protocols employed to improve the management of cancer patients. The journal also focuses on the impact of management programs and new therapeutic agents and protocols on

patient perspectives such as quality of life, adherence and satisfaction. The manuscript management system is completely online and includes a very quick and fair peer-review system, which is all easy to use. Visit http://www.dovepress.com/testimonials.php to read real quotes from published authors.

Submit your manuscript here: http://www.dovepress.com/oncotargets-and-therapy-journal 